\title{
Risk Prediction of Hepatocellular Carcinoma in Patients With Alcoholic Cirrhosis in an Area With Intermediate Prevalence for Hepatitis B Virus Infection: a Competing Risk Analysis
}

\section{Kyunghan Lee}

Seoul National University Bundang Hospital

\section{Gwang Hyeon Choi}

Seoul National University Bundang Hospital

\section{Eun Sun Jang}

Seoul National University Bundang Hospital

\section{Sook-Hyang Jeong}

Seoul National University Bundang Hospital

Jin-Wook Kim ( $\square$ kimjw@snubh.org)

Seoul National University College of Medicine https://orcid.org/0000-0003-0934-3344

\section{Research Article}

Keywords: alcohol, alcoholic cirrhosis, cause-specific hazards, competing risk, cumulative incidence, estimation, HBV, HCV, Subdistribution hazards, surveillance

Posted Date: September 14th, 2021

DOl: https://doi.org/10.21203/rs.3.rs-856622/v1

License: (c) (i) This work is licensed under a Creative Commons Attribution 4.0 International License. Read Full License 


\section{Abstract}

Background \& Aims: The role of hepatocellular carcinoma (HCC) surveillance is being questioned in alcoholic cirrhosis because of the relative low incidence of HCC. Comorbid viral hepatitis may modify the risk, and competing outcomes may influence the actual incidence of HCC in alcoholic cirrhosis. This study aimed to assess the risk and predictors of HCC in patients with alcoholic cirrhosis by using competing risk analysis in an area with intermediate prevalence for hepatitis B virus.

Methods: A total of 965 patients with alcoholic cirrhosis were recruited at a university-affiliated hospital in Korea and randomly assigned to either the derivation $(n=643)$ and validation $(n=322)$ cohort.

Subdistribution hazards model of Fine and Gray was used with deaths and liver transplantation treated as competing risks. Death records were confirmed from Korean government databases. A nomogram was developed to calculate the Alcohol-associated Liver Cancer Estimation (ALICE) score.

Results: Markers for viral hepatitis were positive in $21.0 \%$ and $25.8 \%$ of patients in derivation and validation cohort, respectively. The cumulative incidence of HCC was 13.5 and $14.9 \%$ at 10 years for derivation and validation cohort, respectively. Age, positivity for viral hepatitis markers, alpha-fetoprotein level, and platelet count were identified as independent predictors of HCC and incorporated in the ALICE score, which discriminated low, intermediate, and high risk for HCC in alcoholic cirrhosis at the cut-off of 120 and 180.

Conclusions: ALICE score reliably stratifies HCC risk of alcoholic cirrhosis in an area where the prevalence of viral hepatitis is substantial.

\section{Introduction}

Alcohol-related liver disease (ALD) poses great global health burden. According to the Global Burden of Disease study 2017, 332,300 people died of ALD annually, which comprises approximately one fourth of mortalities associated with chronic liver disease [1]. Hepatocellular carcinoma (HCC), the most common form of primary liver cancer in ALD, is responsible for one-third of ALD-related mortality, and one-third of all HCC-related deaths are attributed to alcohol use globally [2]. Surveillance for HCC is recommended for high-risk groups in order to facilitate early detection and improve survival [3]. However, alcohol-related $\mathrm{HCC}$ is prone to insufficient surveillance and therefore delayed detection compared to viral hepatitisassociated HCC [4]. One of the reasons for under-surveillance may be related to relatively low incidence of HCC in ALD. For example, a recent Swedish cohort study $(n=3,410)$ reported HCC incidence rate of 6.2 per 1000 person-years and the 10-year cumulative incidence of only $5.0 \%$ in alcoholic cirrhosis [5], which was much lower than previously published (annual incidence of 2.6-2.9\%) [6-9]. Another recent Danish study showed similar result (cumulative incidence of $6.0 \%$ after 10 years) [10]. These findings suggest that HCC screening for all alcoholic cirrhosis may not be cost-effective, and that further risk stratification is warranted to identify ideal candidates for surveillance in alcoholic cirrhosis. 
Risk stratification strategies have been actively explored in chronic viral hepatitis [11, 12]. However, risk prediction models in ALD have been less well known [13-15]. In building a HCC prediction model, deaths and liver transplantations should be considered as competing events because many ALD patients experience hepatic decompensations and deaths before HCC is detected. Conventional Kaplan-Meier and Cox analysis may over-estimate the actual risk of HCC in the presence of competing risks [16]. For competing-risk survival analysis, cause-specific hazards or Fine-Gray model is recommended [17]. The aforementioned alcohol-related HCC prediction models, however, used conventional cox regression without competing risk analysis.

Hepatitis $B$ virus (HBV) and hepatitis $C$ virus ( $\mathrm{HCV}$ ) may accelerate disease progression and increase the HCC risk in ALD [18]. Therefore, comorbid viral hepatitis needs to be considered in the generation of prognostic models for ALD. However, the effect of hepatitis virus infection has not been thoroughly assessed because patients with chronic viral hepatitis were usually excluded in the epidemiologic studies of alcoholic cirrhosis $[9,10,15]$. The prevalence of HBV is decreasing over time in Korea, but still is classified as intermediate, i.e., $\geq 2 \%$ of population [19].

In this study, we aimed to assess the risk and predictors of HCC in Korean alcoholic cirrhosis patients by using competing risk analysis. For this aim, we linked the Korean national death registry data to hospitalbased cohort data. A HCC risk prediction nomogram was developed, taking into consideration liver transplantations and deaths as competing risks.

\section{Methods}

\section{Study population and design}

In this retrospective cohort study, an e-cohort was generated by using the clinical data warehouse of Seoul National University Bundang Hospital, a university-affiliated hospital in Korea [20-22]. The inclusion criteria were: 1) ALD based on ICD-10 code K70 AND presence of cirrhosis (see below), 2) > 20 years of age, 3) screening liver ultrasonography (US) with or without serum alpha-fetoprotein (AFP) at baseline. Alcoholic cirrhosis was diagnosed based on histology, endoscopic confirmation of varices or radiologic demonstration of cirrhosis. Patients were excluded if follow-up duration was $<6$ months, development of outcomes (see below) or other malignancies before or within 6 months from initial screening US. Child-Pugh class $\mathrm{C}$ patients at presentation were also excluded because HCC surveillance was generally not recommended unless they are on the transplant waiting list $[3,23,24]$.

The primary outcome was development of HCC. Secondary outcomes were liver transplantation and death which were assessed as competing risks. The death records were confirmed by using the Korean government database of vital statistics generated by Statistics Korea and Ministry of the Interior and Safety.

\section{HCC surveillance}


HCC surveillance was performed by liver US with or without serum AFP at 6-12 months of interval at the discretion of the attending hepatologists. Multiphase CT or MRI were subsequently performed if liver US exam showed nodule(s) with a diameter $\geq 10 \mathrm{~mm}$, or portal vein thrombosis, or increased AFP level. The diagnosis of hepatocellular carcinoma (HCC) was confirmed based on LiRAD 5 criteria [25]. Liver biopsy was performed to make a definitive diagnosis if imaging studies showed atypical findings [23].

This study was approved by the institutional review board (IRB) and ethics committee of SNUBH (IRB No: B-1907-553-105). All clinical investigations have been conducted according to the principles expressed in the Declaration of Helsinki. The requirement of informed consent was waived due to the retrospective nature of this study and anonymous analysis of data.

\section{Statistical analysis}

Enrolled patients were randomly assigned to one of two cohorts in a 2:1 ratio: the derivation and validation cohorts. Competing risk regression models were used with deaths and liver transplantation being treated as competing risks to assess the absolute risk of $\mathrm{HCC}$ and to identify the predictors of alcohol-related HCC from the derivation cohort. For competing risk analysis, the cause-specific cumulative incidences were plotted by non-parametric cumulative incidence function using STATA's stcurve cif, and the subdistribution hazards model of Fine and Gray was built by using STATA's stcrreg competing-risks regression [26, 27]. Complete case analysis method was chosen for handling missing data. A nomogram was developed for calculating the HCC scoring system by using R rms package. The calibration of the scoring system was evaluated by using calibration curves ( $\mathrm{R}$ riskRegression package). The predictive power of the scoring system was compared by using area under time-dependent ROC analysis with $\mathrm{R}$ timeROC package. Continuous variables were expressed as their median values and interquartile range (IQR), and compared using Wilcoxon rank sum test. Categorical variables were expressed as percentages, and compared using chi-square test. All statistical analyses were performed using STATA for windows ver. 14 (STATA corp., Texas, USA) and R statistical package ver. 3.6.1 (The R Foundation for Statistical Computing, Vienna, Austria; http://R-project.org).

\section{Results}

\section{Baseline characteristics of study cohorts}

We identified 4,980 patients with ALD who visited our institution and received screening US between April 1, 2004 and December 31, 2017. Among them, 965 patients with alcoholic cirrhosis were finally included in this study and randomly allocated to either derivation $(n=643)$ or validation cohort $(n=322)$. The baseline characteristics of the two cohorts were balanced without significant differences except for baseline AFP levels (Table 1). The markers for viral hepatitis were positive in 21.0 and $25.8 \%$ of patients in derivation and validation cohort, respectively $(p=0.106)$. 
Table 1

Baseline characteristics of patients with alcoholic cirrhosis.

\begin{tabular}{|c|c|c|c|}
\hline & Derivation cohort $(n=643)$ & Validation cohort $(n=322)$ & $P$ value \\
\hline Age (year) & $55(15)$ & $55(12)$ & 0.364 \\
\hline Male sex (\%) & 80 & 77 & 0.266 \\
\hline Decompensated cirrhosis (\%) & 37 & 33 & 0.137 \\
\hline Diabetes (\%) & 31 & 30 & 0.832 \\
\hline Hypertension (\%) & 21 & 23 & 0.589 \\
\hline Dyslipidemia (\%) & 33 & 32 & 0.796 \\
\hline $\mathrm{BMI}\left(\mathrm{kg} / \mathrm{m}^{2}\right)$ & $25.3(4.8)$ & $25.2(5.9)$ & 0.745 \\
\hline Alcohol use (g/day) & $54(89)$ & $54(85)$ & 0.972 \\
\hline Duration of alcohol use $(y)$ & $30(15)$ & $30(20)$ & 0.598 \\
\hline HBsAg positivity (\%) & 19 & 21 & 0.464 \\
\hline Anti-HCV positivity (\%) & 3.4 & 5.0 & 0.242 \\
\hline $\operatorname{AFP}(\mathrm{ng} / \mathrm{mL})$ & $3.7(3.2)$ & $4.1(3.6)$ & 0.015 \\
\hline AST (IU/L) & $45(51)$ & $46(48)$ & 0.671 \\
\hline ALT (IU/L) & $38(42)$ & $36(40)$ & 0.703 \\
\hline Prothrombin time (INR) & $1.08(0.17)$ & $1.08(0.18)$ & 0.955 \\
\hline Platelet count $\left(\times 10^{3} / \mathrm{mm}^{3}\right)$ & $173(107)$ & $173(107)$ & 0.822 \\
\hline Total bilirubin $(\mathrm{mg} / \mathrm{dL})$ & $1.0(0.7)$ & $1.0(0.8)$ & 0.154 \\
\hline Albumin (mg/dL) & $4.2(0.7)$ & $4.1(0.7)$ & 0.369 \\
\hline GGT (U/L) & $97(117)$ & $101(280)$ & 0.176 \\
\hline $\operatorname{ALP}(U / L)$ & $90(57)$ & $91(57)$ & 0.801 \\
\hline Child-Pugh class A / B (\%) & $70 / 30$ & $72 / 30$ & 0.439 \\
\hline
\end{tabular}

Continuous variables were expressed as their median values (interquartile range), and p-value was calculated using Wilcoxon rank-sum test. Categorical variables were expressed as absolute numbers (percentages), and p-value was calculated using chi-square test.

FIB-4 index[37] = age (yr) $\square$ AST $(\mathrm{U} / \mathrm{L}) /$ Platelet count $\left(10^{9} / \mathrm{L}\right) \rrbracket(\mathrm{ALT}(\mathrm{U} / \mathrm{L}))^{0.5}$

$\mathrm{BMI}$, body mass index; HbsAg, hepatitis B surface antigen; Anti-HCV, antibody against hepatitis C virus; AFP, alpha-fetoprotein; AST, aspartate aminotransferase; ALT, alanine aminotransferase; INR, international normalized ratio; GGT, gamma-glutamyltransferase; ALP, alkaline phosphatase 


\begin{tabular}{|c|c|c|c|}
\hline & Derivation cohort $(n=643)$ & Validation cohort $(n=322)$ & $P$ value \\
\hline FIB-4 index* & $2.56(3.40)$ & $2.44(4.63)$ & 0.872 \\
\hline Liver stiffness value, $\mathrm{kPa}$ & $7.9(8.7)$ & $8.7(8.4)$ & 0.732 \\
\hline \multicolumn{4}{|c|}{$\begin{array}{l}\text { Continuous variables were expressed as their median values (interquartile range), and p-value was } \\
\text { calculated using Wilcoxon rank-sum test. Categorical variables were expressed as absolute numbers } \\
\text { (percentages), and p-value was calculated using chi-square test. }\end{array}$} \\
\hline \multicolumn{4}{|c|}{ FIB-4 index[37] = age $(\mathrm{yr}) \square \mathrm{AST}(\mathrm{U} / \mathrm{L}) /$ Platelet count $\left(10^{9} / \mathrm{L}\right) \otimes(\mathrm{ALT}(\mathrm{U} / \mathrm{L}))^{0.5}$} \\
\hline \multicolumn{4}{|c|}{$\begin{array}{l}\text { BMI, body mass index; HbsAg, hepatitis B surface antigen; Anti-HCV, antibody against hepatitis C } \\
\text { virus; AFP, alpha-fetoprotein; AST, aspartate aminotransferase; ALT, alanine aminotransferase; INR, } \\
\text { international normalized ratio; GGT, gamma-glutamyltransferase; ALP, alkaline phosphatase }\end{array}$} \\
\hline
\end{tabular}

\section{Incidence of HCC in alcoholic cirrhosis}

During the median follow-up period of 63 months (range 6-197), 102 patients developed HCC, 8 received liver transplantation, and 224 patients died without HCC. The cumulative HCC incidence was $7.1 \%$ and $7.8 \%$ at 5 years, and 13.5 and $14.9 \%$ at 10 years for derivation and validation cohort, respectively (Fig. 1). If patients with positive viral hepatitis markers were excluded, the cumulative HCC incidence decreased to $5.5 \%$ and $5.1 \%$ at 5 years, and $10.3 \%$ and $9.6 \%$ at 10 years for derivation and validation cohort, respectively.

\section{Predictors of HCC in alcoholic cirrhosis}

Univariate subdistribution hazards model analysis of the derivation cohort demonstrated that older age, male sex, chronic viral hepatitis, higher AFP level, and lower platelet counts were significantly associated with increased risk of HCC. Among them, four predictors were independently identified through multivariate analysis: age, positive for viral hepatitis markers, AFP level, and platelet count (Table 2). 
Table 2

Predictors for HCC development by Fine and Gray's proportional subhazards model in derivation cohort $(n=483)$

\begin{tabular}{|c|c|c|c|c|}
\hline \multirow[t]{2}{*}{ Variables } & \multicolumn{2}{|c|}{ Univariate } & \multicolumn{2}{|l|}{ Multivariate } \\
\hline & $\begin{array}{l}\text { Subhazard ratio }(95 \% \\
\mathrm{Cl})\end{array}$ & $P$ value & $\begin{array}{l}\text { Subhazard ratio }(95 \% \\
\mathrm{Cl})\end{array}$ & $\begin{array}{l}P \\
\text { value }\end{array}$ \\
\hline Age $(y)$ & $1.02(1.00-1.04)$ & 0.03 & $1.03(1.01-1.05)$ & $<0.01$ \\
\hline Male sex & $2.58(1.03-6.44)$ & 0.04 & $2.48(0.99-6.22)$ & 0.05 \\
\hline Diabetes & $0.83(0.40-1.72)$ & 0.62 & & \\
\hline Hypertension & $1.35(0.81-2.26)$ & 0.25 & & \\
\hline Dyslipidemia & $1.20(0.73-1.97)$ & 0.47 & & \\
\hline $\mathrm{BMI}\left(\mathrm{kg} / \mathrm{m}^{2}\right)$ & $1.03(0.98-1.09)$ & 0.22 & & \\
\hline Alcohol use (g/day) & $1.00(1.00-1.00)$ & 0.86 & & \\
\hline $\begin{array}{l}\text { Duration of alcohol use } \\
\text { (y) }\end{array}$ & $1.01(0.98-1.03)$ & 0.61 & & \\
\hline HBV/HCV positive ${ }^{a}$ & $1.71(1.02-2.85)$ & 0.04 & $1.80(1.03-3.12)$ & 0.04 \\
\hline $\operatorname{AFP}(\mathrm{ng} / \mathrm{mL}, \log 10)$ & $2.30(1.48-3.57)$ & $<0.01$ & $1.76(1.10-2.82)$ & 0.02 \\
\hline AST (IU/L) & $1.00(1.00-1.00)$ & 0.19 & & \\
\hline ALT (IU/L) & $1.00(1.00-1.00)$ & 0.53 & & \\
\hline Prothrombin time (INR) & $1.70(0.93-3.13)$ & 0.08 & & \\
\hline $\begin{array}{l}\text { Platelet count } \\
\left(x 10^{3} / \mathrm{mm}^{3}\right)\end{array}$ & $0.99(0.99-1.00)$ & $<0.01$ & $0.99(0.99-1.00)$ & 0.02 \\
\hline Total bilirubin (mg/dL) & $0.99(0.90-1.10)$ & 0.90 & & \\
\hline Albumin (mg/dL) & $0.66(0.44-1.00)$ & 0.05 & & \\
\hline GGT (U/L) & $1.00(1.00-1.00)$ & 0.14 & & \\
\hline $\operatorname{ALP}(U / L)$ & $1.00(1.00-1.00)$ & 0.99 & & \\
\hline
\end{tabular}

\section{Development and validation of alcohol-associated liver cancer estimation (ALICE) scoring system}

A parsimonious HCC prediction model, the alcohol-associated liver cancer estimation (ALICE) scoring system, was developed from the result of multivariate cumulative incidence function. Nomogram was 
constructed with four predictors to calculate the ALICE score (Fig. 2). The calibration plots of the nomogram showed good agreement between the observed and predicted HCC risks (Supplementary Fig. 1). When patients were stratified by ALICE score, HCC risk was minimal with a cut-off $\leq 120$, whereas patients with a cut-off of $>120$ and $<180$ showed the cumulative incidence exceeding $15 \%$ per 10 years, and patients with $\geq 180$ had highest risk for HCC (Fig. 3 and Table 3).

Table 3

Estimated cumulative incidence of HCC according to ALICE score

\begin{tabular}{|lll|}
\hline ALICE score & Derivation cohort & Validation cohort \\
\hline & 5 -year HCC risk & \\
$<=120$ & 0.9 & 1.8 \\
$>120$ and $<=180$ & 7.2 & 7.0 \\
$>180$ & 31.5 & 33.8 \\
& 10 -year HCC risk & \\
$<=120$ & 1.3 & 2.8 \\
$>120$ and $<=180$ & 17.0 & 16.6 \\
$>180$ & 37.2 & 39.9 \\
\hline
\end{tabular}

Finally, we compared the predictive performance of ALICE score with that of the model derived from US Veterans Affairs health care system (US-VA model), which is a internally validated scoring system for predicting the risk of HCC in patients with alcoholic cirrhosis by using age, sex, BMI, diabetes, platelet count, serum albumin, and serum AST/ ALT ratio as predictors [15]. Time-dependent ROC curve analysis revealed that the performance of ALICE score had comparable or higher AUC values than UA-VA score in the validation cohort (Fig. 4).

\section{Discussion}

In this study, we described HCC risk in alcoholic liver cirrhosis, and developed a risk stratification model for HCC (i.e., ALICE score) in a hospital-based cohort. Unlike the recently developed prediction models [14, 15], we employed competing-risk analysis by incorporating mortality data from causes other than HCC. Liver cirrhosis is typically a multistate disease complicated by discrete outcomes [28]. If patients with competing outcomes such as non-HCC deaths are simply treated as right-censored cases, Kaplan Meier method may overestimate the real cumulative risks $[28,29]$. Moreover, the predicted risk of HCC does not necessarily correlate with the predicted rate by Cox model of HCC prediction [29]. Our cohort patients showed that censored cases due to non-HCC deaths were twice more than those censored due to HCC. 
The estimated cumulative HCC risk in our cohort was $1.5 \%$ per year for overall patients (Fig. 1), and approximately $1.0 \%$ for patients without markers of viral hepatis. The latter figure fell in the range between the two recent European studies (0.7 [10] and $1.8 \%$ [9]) which excluded patients with chronic viral hepatitis. Comorbid viral hepatitis is of special interest in geographic areas whe re chronic hepatitis virus infection is prevalent. Interestingly, our cohort showed higher prevalence of chronic viral hepatitis compared to Korean general population: $2.9 \%$ for HBV [19] and 0.8 \% for HCV [30]. This high prevalence may be explained by the synergistic effect of comorbid viral hepatitis on the accelerated progression of alcoholic fatty liver to alcoholic cirrhosis [31].

The role of HCC surveillance in alcoholic liver disease is still under debate. Practice guidelines recommend HCC surveillance in patients with cirrhosis due to alcohol and other etiologies on the ground that threshold HCC incidence of $>1.5 \%$ /year may justify cost-effectiveness of surveillance [3, 23, 32]. However, not only the " $1.5 \%$ /year" cut-off itself has been doubted [33], but also the risk of HCC in alcoholic cirrhosis may not be high enough to ensure cost-effectiveness $[5,10]$. Risk stratification may be thus necessary to enhance the effectiveness of HCC surveillance in alcoholic cirrhosis.

We have built our risk stratification model based on four independent predictors of HCC risk: age, chronic viral hepatitis, AFP level, and platelet count. AFP level was a significant predictor in addition to other wellestablished markers [14, 15], and this finding is in concordance with the French cohort study [9]. These four factors are readily available in routine practice, and nomogram-based ALICE score was able to discriminate the low, high, and super high-HCC risk groups in alcoholic cirrhosis. Patients with ALICE score $\leq 120$ carries minimal risk for HCC and may not be indicated for routine HCC surveillance, whereas those with $\geq 180$ show highest risk for HCC and regular surveillance may be justified. In other word, the ALICE score may serve dual purposes: (1) to exclude ALD patients with low risk from HCC surveillance, and (2) to identify patients with very high risk for HCC in need of enhanced surveillance. Further studies will be necessary to assess whether risk-based surveillance is cost-effective in alcoholic cirrhosis.

Recently, a HCC risk stratification model was developed from US Veterans Affairs healthcare system (VAHS) data including a large number of alcoholic cirrhosis $(n=16,175)$ [15]. The model was based on 8 parameters and the score is available through a web-based calculator. As mentioned above, competing risks were not considered in the US-VA model building. Moreover, a significant fraction of alcoholic cirrhosis with viral markers will not be properly assessed by the US-VA model. Time-dependent ROC analysis showed that the ALICE score had comparable or higher AUC values compared with the US-VA score (Fig. 4). Compared to the US-VA model, our score is more parsimonious with using only 4 readily available parameters. However, further validation would be warranted for the clinical utility of ALICE score by prospective studies.

There are potential limitations in our study, mainly related to the retrospective design in a limited number of institutions. We tried to minimize selection bias by using our pre-defined EMR system[20,34] and validated the model in an internal validation cohort; however, further external validation is needed by prospective studies. Cost-effectiveness analysis should also be conducted for the clinical utility of ALICE 
score-guided surveillance strategy. Finally, the diagnosis of cirrhosis was mostly made clinically, and there was a possibility that liver cirrhosis was underdiagnosed and not included in our cohort [35, 36]. Since liver biopsy is not generally required for the management of compensated alcoholic liver disease, however, we believe that our model can be applicable to real-world practice of clinically diagnosed alcoholic liver cirrhosis.

In conclusion, a novel HCC risk score, the ALICE score, which includes age, chronic viral hepatitis, AFP level, and platelet count, represents a reliable and easy-to-use method for predicting HCC development in patients with alcoholic cirrhosis in areas where the prevalence of viral hepatitis is substantial.

\section{Abbreviations}

AFP, alpha-fetoprotein; ALICE, alcohol-associated liver cancer estimation; ALD, Alcohol-related liver disease; ALP, alkaline phosphatase; ALT, alanine aminotransferase; AST, aspartate aminotransferase; AUC, area under the curve; BCLC, Barcelona Clinic Liver Cancer; GGT, gamma-glutamyl transferase; HBV; $B M I$, body mass index; hepatitis B virus; HCC, hepatocellular carcinoma; HCV, hepatitis C virus; ICD, International Classification of Disease; IQR, interquartile range; ROC, receiver operating characteristic; INR, international normalized ratio; NAFLD, non-alcoholic fatty liver disease; US, ultrasonography;

\section{Declarations}

\section{Conflicts of interest}

Kyunghan Lee, Gwang Hyeon Choi, Eun Sun Jang, Sook-Hyang Jeong, and Jin-Wook Kim declare that they have no conflict of interest.

\section{Financial support:}

This work was supported by a National Research Foundation of Korea (NRF) grant to J-W Kim, funded by the Korean Government (2017R1D1A1B03031483). The funders had no role in the study design, data collection and analysis, decision to publish, or preparation of the manuscript.

\section{Ethics approval}

The IRB approved the study protocol (IRB No: B-1907-553-105).

\section{Consent to participate}

Written consents were waived by the IRB due to the retrospective nature of study.

\section{Consent for publication}

All authors agree to publication if the paper is accepted. 
Availability of data and material

Data will be shared on request to the corresponding author with permission of our IRB.

\section{Code availability}

Not applicable

\section{Animal research}

Not applicable

\section{Clinical trials registration}

Not applicable

Gels and Blots/ Image Manipulation

Not applicable

\section{AUTHORSHIP}

Guarantor of the article: Jin-Wook Kim.

\section{Author contributions:}

Jin-Wook Kim: designed the research study, collected and analyzed the data and wrote the paper;

Kyunghan Lee: collected and analyzed the data and wrote the paper; Gwang Hyeon Choi, Eun Sun Jang, Sook-Hyang Jeong: analyzed the data and wrote the paper.

\section{ORCID}

Jin-Wook Kim http://orcid.org/0000-0003-0934-3344

Kyunghan Lee https://orcid.org/0000-0001-7605-0782

Eun Sun Jang https://orcid.org/0000-0003-4274-2582

Sook Hyang Jeong https://orcid.org/0000-0002-4916-7990

Gwang Hyeon Choi http://orcid.org/0000-0002-8795-8427

\section{References}

1. Collaborators GBDCoD. Global, regional, and national age-sex-specific mortality for 282 causes of death in 195 countries and territories, 1980-2017: a systematic analysis for the Global Burden of 
Disease Study 2017. Lancet. 2018;392:1736-88.

2. Global Burden of Disease Liver. Cancer C, Akinyemiju T, Abera S, Ahmed M, Alam N, Alemayohu MA, Allen C, et al. The Burden of Primary Liver Cancer and Underlying Etiologies From 1990 to 2015 at the Global, Regional, and National Level: Results From the Global Burden of Disease Study 2015. JAMA Oncol 2017;3:1683-91.

3. European Association for the Study of the Liver. Electronic address eee, European Association for the Study of the L. EASL Clinical Practice Guidelines: Management of hepatocellular carcinoma. J Hepatol. 2018;69:182-236.

4. Ganne-Carrie N, Nahon P. Hepatocellular carcinoma in the setting of alcohol-related liver disease. J Hepatol. 2019;70:284-93.

5. Hagstrom H, Thiele M, Sharma R, Simon TG, Roelstraete B, Soderling J, Ludvigsson JF. Risk of Cancer in Biopsy-Proven Alcohol-Related Liver Disease: A Population-Based Cohort Study of 3410 Persons. Clinical gastroenterology and hepatology: the official clinical practice journal of the American Gastroenterological Association 2021.

6. Joshi K, Kohli A, Manch R, Gish R. Alcoholic Liver Disease: High Risk or Low Risk for Developing Hepatocellular Carcinoma? Clin Liver Dis. 2016;20:563-80.

7. Mancebo A, Gonzalez-Dieguez ML, Cadahia V, Varela M, Perez R, Navascues CA, Sotorrios NG, et al. Annual incidence of hepatocellular carcinoma among patients with alcoholic cirrhosis and identification of risk groups. Clinical gastroenterology hepatology: the official clinical practice journal of the American Gastroenterological Association. 2013;11:95-101.

8. Peter Jepsen M, Peter Ott PhD. MD, DMSc; Per Kragh Andersen, PhD, DMSc; Henrik Toft Sørensen, MD, PhD, DMSc; and Hendrik Vilstrup, MD, DSc. Risk for Hepatocellular Carcinoma in Patients With Alcoholic Cirrhosis. Ann Intern Med. 2012;156.

9. Ganne-Carrie N, Chaffaut C, Bourcier V, Archambeaud I, Perarnau JM, Oberti F, Roulot D, et al. Estimate of hepatocellular carcinoma incidence in patients with alcoholic cirrhosis. J Hepatol. 2018;69:1274-83.

10. Jepsen P, Kraglund F, West J, Villadsen GE, Sorensen HT, Vilstrup H. Risk of hepatocellular carcinoma in Danish outpatients with alcohol-related cirrhosis. J Hepatol. 2020;73:1030-6.

11. Varbobitis I, Papatheodoridis GV. The assessment of hepatocellular carcinoma risk in patients with chronic hepatitis B under antiviral therapy. Clin Mol Hepatol. 2016;22:319-26.

12. Ioannou GN, Green PK, Beste LA, Mun EJ, Kerr KF, Berry K. Development of models estimating the risk of hepatocellular carcinoma after antiviral treatment for hepatitis C. J Hepatol. 2018;69:1088-98.

13. Flemming JA, Yang JD, Vittinghoff E, Kim WR, Terrault NA. Risk prediction of hepatocellular carcinoma in patients with cirrhosis: the ADRESS-HCC risk model. Cancer. 2014;120:3485-93.

14. Sharma SA, Kowgier M, Hansen BE, Brouwer WP, Maan R, Wong D, Shah H, et al. Toronto HCC risk index: A validated scoring system to predict 10-year risk of HCC in patients with cirrhosis. J Hepatol 2017. 
15. Ioannou GN, Green P, Kerr KF, Berry K. Models estimating risk of hepatocellular carcinoma in patients with alcohol or NAFLD-related cirrhosis for risk stratification. J Hepatol. 2019;71:523-33.

16. Wolkewitz M, Cooper BS, Bonten MJ, Barnett AG, Schumacher M. Interpreting and comparing risks in the presence of competing events. BMJ. 2014;349:g5060.

17. Zhang Z, Cortese G, Combescure C, Marshall R, Lee M, Lim HJ, Haller B, et al. Overview of model validation for survival regression model with competing risks using melanoma study data. Ann Transl Med. 2018;6:325.

18. Matsushita H, Takaki A. Alcohol and hepatocellular carcinoma. BMJ Open Gastroenterol. 2019;6:e000260.

19. Yim SY, Kim JH. The epidemiology of hepatitis B virus infection in Korea. Korean J Intern Med. 2019;34:945-53.

20. Yoo S, Lee KH, Lee HJ, Ha K, Lim C, Chin HJ, Yun J, et al. Seoul National University Bundang Hospital's Electronic System for Total Care. Healthcare informatics research. 2012;18:145-52.

21. Chung JW, Kim BH, Lee CS, Kim GH, Sohn HR, Min BY, Song JC, et al. Optimizing Surveillance Performance of Alpha-Fetoprotein by Selection of Proper Target Population in Chronic Hepatitis B. PLoS One. 2016;11:e0168189.

22. Yoo S, Hwang $H$, Jheon S. Hospital information systems: experience at the fully digitized Seoul National University Bundang Hospital. J Thorac Dis. 2016;8:637-41.

23. Marrero JA, Kulik LM, Sirlin CB, Zhu AX, Finn RS, Abecassis MM, Roberts LR, et al. Diagnosis, Staging, and Management of Hepatocellular Carcinoma: 2018 Practice Guidance by the American Association for the Study of Liver Diseases. Hepatology. 2018;68:723-50.

24. Heimbach JK, Kulik LM, Finn RS, Sirlin CB, Abecassis MM, Roberts LR, Zhu AX, et al. AASLD guidelines for the treatment of hepatocellular carcinoma. Hepatology. 2018;67:358-80.

25. Tang A, Bashir MR, Corwin MT, Cruite I, Dietrich CF, Do RKG, Ehman EC, et al. Evidence Supporting LIRADS Major Features for CT- and MR Imaging-based Diagnosis of Hepatocellular Carcinoma: A Systematic Review. Radiology. 2018;286:29-48.

26. Fine JP, Gray RJ. A Proportional Hazards Model for the Subdistribution of a Competing Risk. J Am Stat Assoc. 1999;94:496-509.

27. Lambert PC. The estimation and modelling of cause-specific cumulative incidence functions using time-dependent weights. Stata J. 2017;17:181-207.

28. D'Amico G, Morabito A, D'Amico M, Pasta L, Malizia G, Rebora P, Valsecchi MG. Clinical states of cirrhosis and competing risks. J Hepatol. 2018;68:563-76.

29. Jepsen P, Vilstrup H, Andersen PK. The clinical course of cirrhosis: The importance of multistate models and competing risks analysis. Hepatology. 2015;62:292-302.

30. Jeong SH, Jang ES, Choi HY, Kim KA, Chung W, Ki M. Current status of hepatitis C virus infection and countermeasures in South Korea. Epidemiol Health. 2017;39:e2017017. 
31. O'Shea RS, Dasarathy S, McCullough AJ. Practice Guideline Committee of the American Association for the Study of Liver D, Practice Parameters Committee of the American College of G. Alcoholic liver disease. Hepatology. 2010;51:307-28.

32. Singal AK, Bataller R, Ahn J, Kamath PS, Shah VH. ACG Clinical Guideline: Alcoholic Liver Disease. Am J Gastroenterol. 2018;113:175-94.

33. Jepsen $P$, West J. We need stronger evidence for (or against) hepatocellular carcinoma surveillance. J Hepatol. 2021;74:1234-9.

34. Yoo S, Kim S, Lee KH, Baek RM, Hwang $\mathrm{H}$. A study of user requests regarding the fully electronic health record system at Seoul National University Bundang Hospital. Stud Health Technol Inform. 2013;192:1015.

35. Lackner C, Spindelboeck W, Haybaeck J, Douschan P, Rainer F, Terracciano L, Haas J, et al. Histological parameters and alcohol abstinence determine long-term prognosis in patients with alcoholic liver disease. J Hepatol. 2017;66:610-8.

36. Shah ND, Ventura-Cots M, Abraldes JG, Alboraie M, Alfadhli A, Argemi J, Badia-Aranda E, et al. Alcohol-Related Liver Disease Is Rarely Detected at Early Stages Compared With Liver Diseases of Other Etiologies Worldwide. Clinical gastroenterology hepatology: the official clinical practice journal of the American Gastroenterological Association. 2019;17:2320-9 e2312.

37. Sterling RK, Lissen E, Clumeck N, Sola R, Correa MC, Montaner J. M SS, et al. Development of a simple noninvasive index to predict significant fibrosis in patients with HIV/HCV coinfection. Hepatology. 2006;43:1317-25.

\section{Figures}




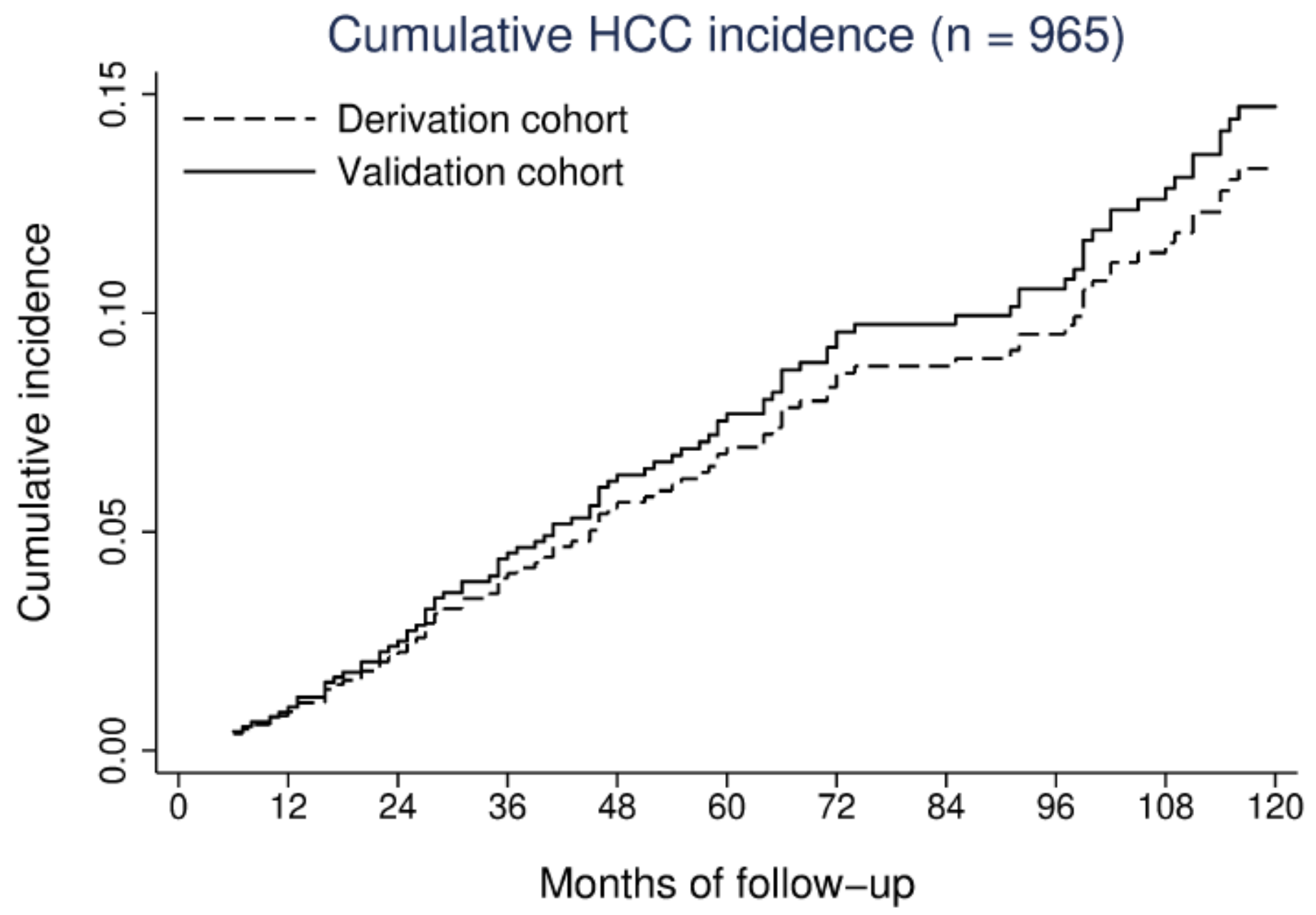

Figure 1

Cumulative incidence functions for HCC in the derivation and validation cohorts. 
Total points

0

$10-20-30$

Age (yr)

Viral hepatitis

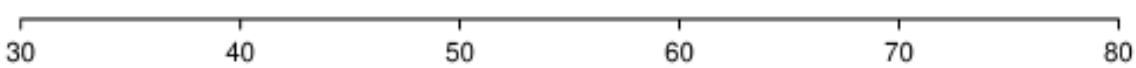

positive

\section{negative}

AFP( $\log \mathrm{ng} / \mathrm{mL})$

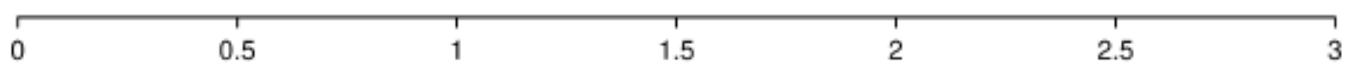

Platelet $(\mathrm{K} / \mathrm{mm} 3)$

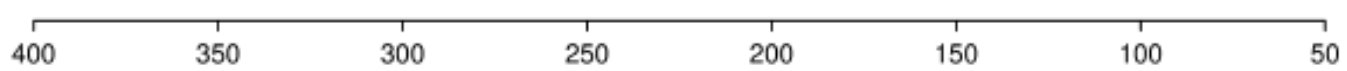

Total Points

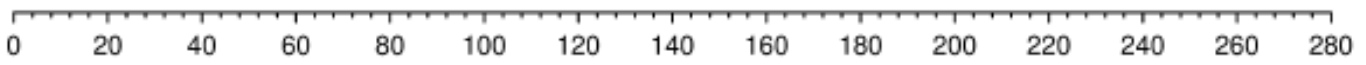

1yr HCC Probability

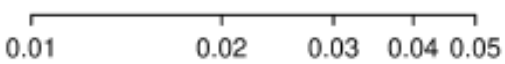

2yr HCC Probability

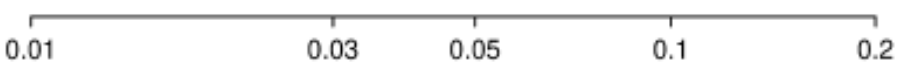

3yr HCC Probability

\begin{tabular}{lllll}
\hline 0.01 & 0.03 & 0.05 & 0.1 & 0.2
\end{tabular}

4yr HCC Probability

\begin{tabular}{llllll}
\hline 0.01 & 0.03 & 0.05 & 0.1 & 0.2 & 0.4
\end{tabular}

\section{Figure 2}

A nomogram for the alcohol-associated liver cancer estimation (ALICE) score. Cumulative incidence curves of HCC in the derivation and validation cohorts according to Alcohol-associated Liver Cancer Estimation (ALICE) score.
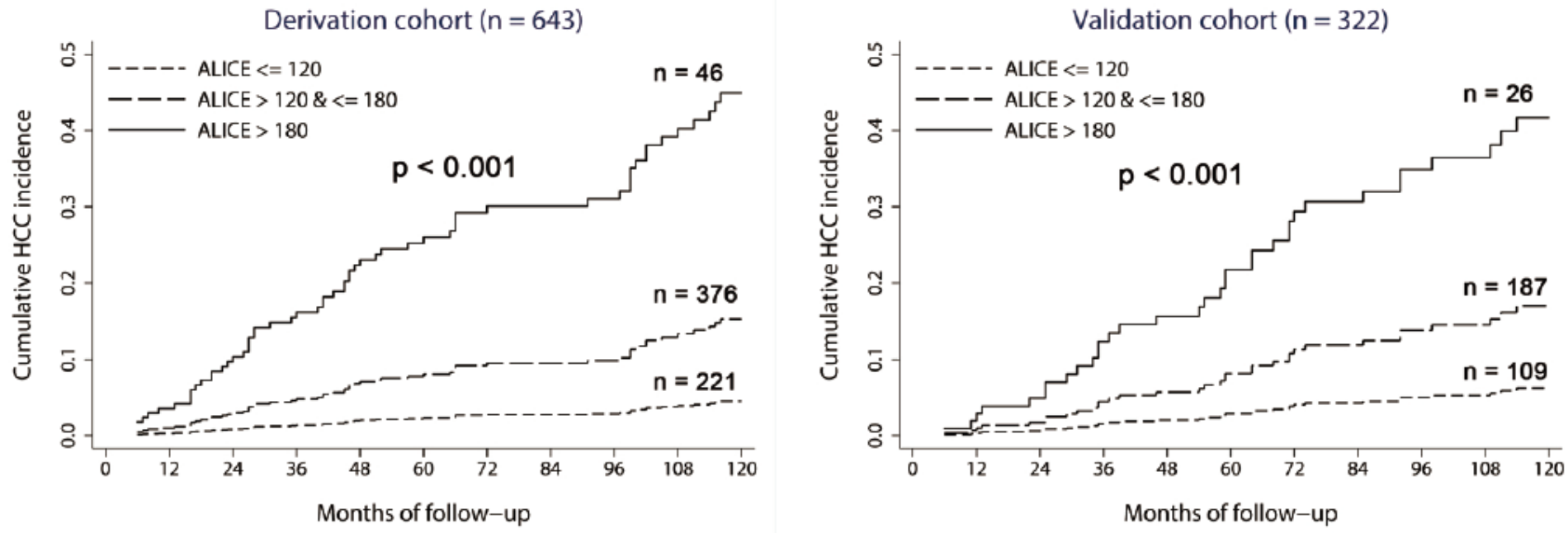

\section{Figure 3}

Cumulative incidence function curves stratified by ALICE score. 
ROC at time $\mathrm{t}=12$

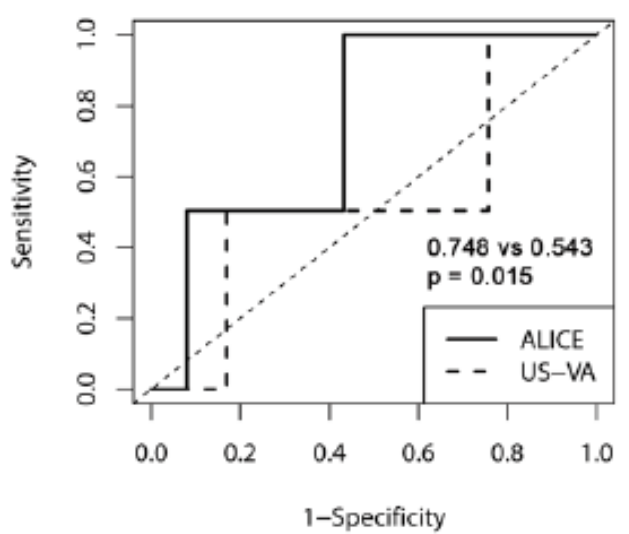

ROC at time $\mathrm{t}=48$

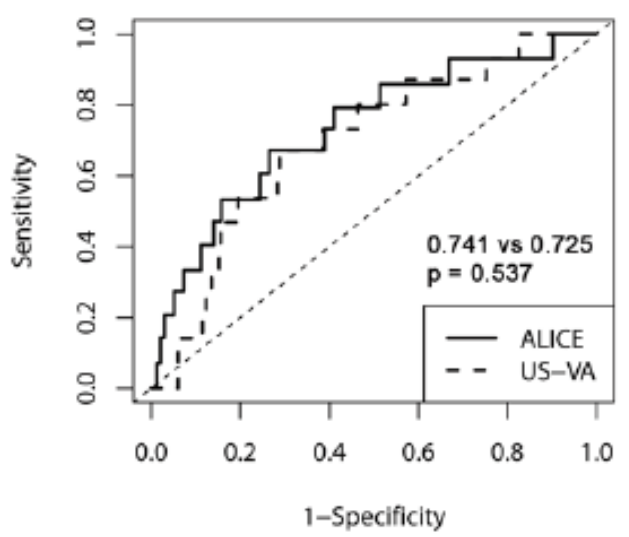

ROC at time $\mathrm{t}=24$

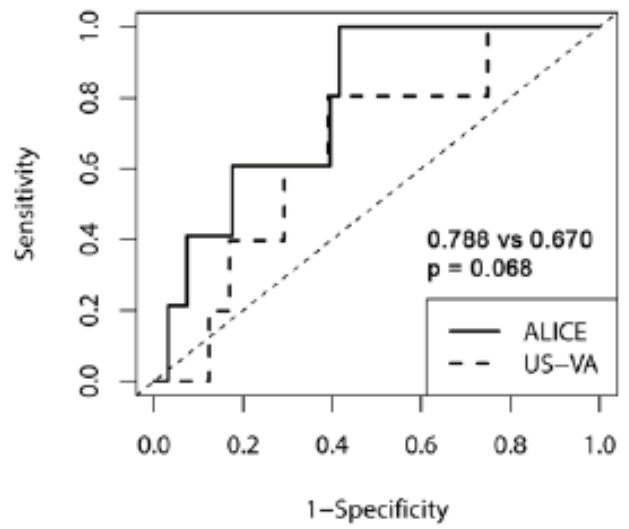

$\mathrm{ROC}$ at time $\mathrm{t}=60$

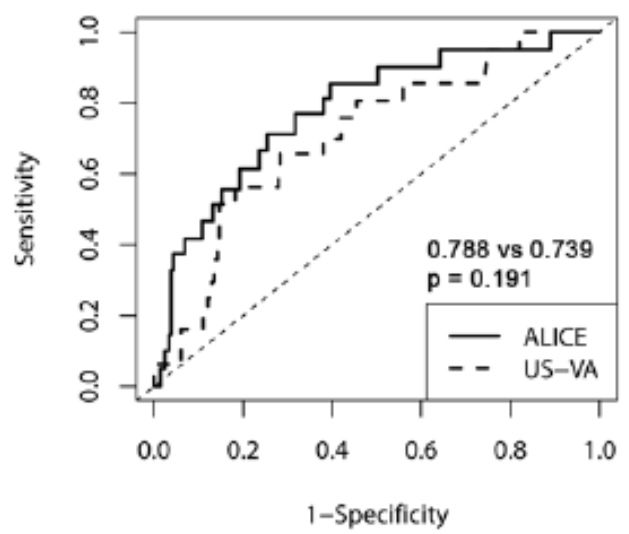

ROC at time $\mathrm{t}=36$

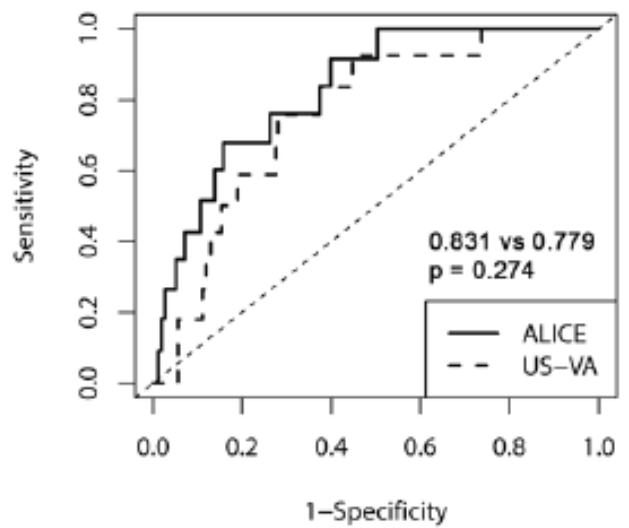

\section{Figure 4}

Comparison of time-dependent receiver operating characteristic curves between the ALICE score and USVA score.

\section{Supplementary Files}

This is a list of supplementary files associated with this preprint. Click to download.

- FigS1calibration.pdf

- floatimage1.jpeg 